\title{
La estrategia de ayuno y realimentación, una alternativa viable para optimizar el consumo de alimento balanceado en el cultivo semi-intensivo de camarón blanco Litopenaeus vannamei
}

\author{
The fasting and refeeding strategy, a viable alternative for optimising the \\ consumption of balanced feed in the semi-intensive farming of white shrimp \\ Litopenaeus vannamei
}

\author{
Junior Jair Castro Morán', Alberto Ordinola-Zapata ${ }^{1,2,3}$
}

\section{Resumen}

\begin{abstract}
La investigación tuvo como objetivo determinar el efecto de dos o tres días de ayuno a la semana con un incremento de $20 \%$ en la ración pos-ayuno en el crecimiento, supervivencia, biomasa, consumo de alimento y factor de conversión relativo (FCR) de camarones Litopenaeus vannamei cultivados en laboratorio a densidad equivalente a la de un sistema semi-intensivo. El experimento se realizó en nueve acuarios con $90 \mathrm{~L}$ de agua salobre, cada uno con ocho camarones de $5.5 \mathrm{~g}$ de peso aproximado $\left(25 \mathrm{ejemplares} / \mathrm{m}^{2}\right)$. Los camarones fueron alimentados dos veces al día, luego se retiraron los residuos de alimento, heces y exuvias. La dosis de alimento suministrado se fijó con base a una tabla comercial y se ajustó de acuerdo con el consumo del día anterior. Durante nueve semanas se evaluó el crecimiento (peso promedio, incremento de peso semanal, ganancia de peso porcentual), supervivencia, biomasa, consumo de alimento y factor de crecimiento relativo - FCR. Los camarones con un ayuno de dos días a la semana y un incremento de $20 \%$ de la ración pos-ayuno mantuvieron un peso y biomasa estadísticamente similar al de camarones sin ayuno, logrando un ahorro de $27.4 \%$ en el alimento suministrado y un mejor FCR (1.18 frente a 1.52). Los camarones con ayuno de tres días a la semana tuvieron
\end{abstract}

${ }^{1}$ Facultad de Ingeniería Pesquera y Ciencias del Mar, Universidad Nacional de Tumbes, Tumbes, Perú

${ }^{2}$ Laboratorio de Acuicultura II, Universidad Nacional de Tumbes, Tumbes, Perú

${ }^{3}$ Email: aordinolaz@untumbes.edu.pe

Recibido: 30 de enero de 2021

Aceptado para publicación: 31 de julio de 2021

Publicado: 27 de octubre de 2021

CLos autores. Este artículo es publicado por la Rev Inv Vet Perú de la Facultad de Medicina Veterinaria, Universidad Nacional Mayor de San Marcos. Este es un artículo de acceso abierto, distribuido bajo los términos de la licencia Creative Commons Atribución 4.0 Internacional (CC BY 4.0) [https:// creativecommons.org/licenses/by/4.0/deed.es] que permite el uso, distribución y reproducción en cualquier medio, siempre que la obra original sea debidamente citada de su fuente original 
un peso promedio y biomasa significativamente inferior, aunque su ahorro en el consumo de alimento y su FCR fueron mejores a los otros dos tratamientos. La supervivencia fue de $100 \%$ en todos los tratamientos. Se concluye que un ayuno de dos días a la semana con un incremento de $20 \%$ de la ración pos-ayuno es viable para optimizar el uso de alimento balanceado en el cultivo de Litopenaeus vannamei en densidad equivalente a la de un cultivo semi-intensivo.

Palabras clave: langostino, peneido, crecimiento compensatorio, restricción alimenticia, engorde

\section{AbSTRACT}

The aim of this study was to determine the effect of two or three days of fasting per week with an increase of $20 \%$ in the post-fasting ration on growth, survival, biomass, feed consumption and relative conversion factor (FCR) of Litopenaeus vannamei shrimp grown in the laboratory at a density equivalent to that of a semi-intensive system. The experiment was carried out in nine aquariums with $90 \mathrm{~L}$ of brackish water, each with eight shrimp weighing approximately $5.5 \mathrm{~g}\left(25 \mathrm{specimens} / \mathrm{m}^{2}\right)$. The shrimp were fed twice a day, then the food residues, faeces and exuviae were removed. The feed dosage was set according to a commercial table and adjusted according to the consumption of the previous day. Growth (average weight, weekly weight increase, percentage weight gain), survival, biomass, feed consumption and relative growth factor - FCR were evaluated for nine weeks. The shrimp fasting two days a week and increasing the post-fasting ration by $20 \%$ maintained a statistically similar weight and biomass to that of shrimp without fasting, achieving a saving of $27.4 \%$ in the feed supplied and a better FCR (1.18 vs. 1.52). Shrimp fasting three days a week had a significantly lower average weight and lower biomass, although their savings in feed consumption and their FCR were better than the other two treatments. Survival was $100 \%$ in all treatments. It is concluded that a two-day weekly fasting with a $20 \%$ increase in the post-fasting ration is feasible to optimize the use of balanced food in the culture of Litopenaeus vannamei at a density equivalent to that of a semi-intensive culture.

Key words: shrimp, penaeid, compensatory growth, feed restriction, fattening

\section{INTRODUCCIÓN}

El cultivo del camarón blanco Litopenaeus vannamei es la mayor actividad de acuicultura de crustáceos a nivel mundial. En 2018 se produjo 4.97 millones de toneladas, que representó el $52.9 \%$ de la producción de crustáceos y $70 \%$ de la producción de camarones peneidos (FAO, 2018, 2020; Bardera et al., 2021). Este cultivo es también una de las actividades acuícolas más importantes en Perú, y la mayor productora de divisas por dicho rubro, toda vez que en 2017 produjo US\$ 215 millones, que representó el 72.6\% de las exportaciones por acuacultura del Perú (Produce, 2018; Tarazona et al., 2018).

Una de las principales limitantes del cultivo de camarón blanco es el alto costo del alimento balanceado, el cual puede significar del 50 al $70 \%$ del costo de producción. Esto es debido al alto nivel de proteína (alrededor de $40 \%$ ) que debe contener el alimento, el cual procede fundamentalmente de la harina de pescado, un insumo con un precio relati- 
vamente alto (Martínez et al., 2013; Faillace et al., 2016; Ghosh, 2018; Hamidoghli et al., 2020). A su vez, los hábitos de alimentación del camarón no permiten el adecuado aprovechamiento del alimento, lo cual a su vez incrementa el nivel de materia orgánica en el agua y el piso de los estanques (Bardera et al., 2021). Se estima que el alimento es el responsable del ingreso en los estanques de cultivo del 76 al $92 \%$ del nitrógeno y 70 a $91 \%$ del fósforo; y que de estos, solo entre 23 a $31 \%$ del nitrógeno y 10 a $13 \%$ del fósforo es incorporado por el camarón (Zhu et al., 2016).

Se han propuesto varias estrategias para optimizar la utilización del alimento balanceado, como el empleo de bandejas de alimentación, alimentadores automáticos, uso de suplementos alimenticios e incluso la modificación de la composición del alimento (Martínez et al., 2013; Ullman et al., 2019; GamboaDelgado et al., 2020). En esta línea, también se viene investigando el empleo de cortos periodos de ayuno (Bezerra et al., 2019).

El ayuno en cultivos de camarón blanco se ha practicado de manera empírica para economizar alimento y mano de obra. De otra parte, tiene como ventaja colateral, que al reducir el consumo de alimento, se reducen las cargas de nutrientes (fósforo y nitrógeno) y de materia orgánica en los efluentes, lo que contribuye a evitar la polución (Maciel et al., 2018). El ayuno se produce habitualmente en el ciclo vital de los organismos acuáticos, pues no siempre pueden encontrar el alimento en el momento que lo necesitan, de allí que estén adaptados para resistir periodos de ayuno (Maciel et al., 2018; Steinberg, 2018). En estos estudios se ha observado que el animal experimenta crecimiento compensatorio, el cual se define como un periodo de rápido crecimiento que se produce cuando un organismo es realimentado luego de un ayuno o una reducción en el suministro de alimento, a fin de restablecer su nivel de desarrollo (Savoie et al., 2017; Liu et al., 2018; Bezerra et al., 2019; Shao et al., 2020).
En diversos organismos acuáticos se ha verificado que se produce crecimiento compensatorio. Entre ellos se tiene a los peces (Oreochromis spp, Sparus aurata, Pangasius bocourti, ciprínidos, Salvelinus fontinalis) (Savoie et al., 2017; Liu et al., 2018; Maciel et al., 2018; Mohanty et al., 2020), moluscos (Octopus mimus, Argopecten irradians, Doryteuthis opalescens, Haliotis asinina, Haliotis midae) (Maciel et al., 2018; Rodríguez-González et al., 2018; Steinberg, 2018), y crustáceos como cangrejos, langostas y camarones (Macrobrachium rosenbergii, Macrobrachium nipponense, Fenneropenaeus chinensis, Astacus leptodactylus, Cherax quadricarinatus, Penaeus monodon) (Mohanty y Mohapatra, 2017; Ghosh et al., 2018; Maciel et al., 2018; Stumpf et al., 2019), y dentro de estos últimos a Litopenaeus vannamei (Zhu et al., 2016; Lara et al., 2017; Maciel et al., 2018). El crecimiento compensatorio se fundamenta en un incremento en la tasa de crecimiento y una mayor eficiencia en la ganancia de peso y se debe a un ajuste fisiológico que ayuda a los organismos a adaptarse para sobrevivir ante eventos adversos en el ambiente como son las altas densidades de cultivo, la hipoxia, el estrés térmico y la restricción alimenticia, entre otras (Lara et al., 2017; Maciel et al., 2018).

Se ha estimado que mediante el crecimiento compensatorio es posible ahorrar alrededor de $25 \%$ del alimento utilizado en el cultivo de camarones Litopenaeus vannamei (Fóes et al., 2016), lo cual generaría un impacto positivo en la rentabilidad de las empresas que se dedican a dicho cultivo, así como una reducción de los niveles de emisión de poluentes de los efluentes de los campos de cultivo. Sin embargo, los resultados han sido contradictorios, pues mientras algunos afirman que es una estrategia factible, otros lo niegan (Steinberg, 2018), posiblemente debido a los sistemas de cultivo empleados, los cuales utilizan diversas densidades de cultivo (Bardera et al., 2021). En una reciente in- 
Cuadro 1. Tratamientos empleados en el experimento

\begin{tabular}{lccc}
\hline \multirow{2}{*}{ Tratamiento } & \multicolumn{2}{c}{ Ayuno semanal } & $\begin{array}{c}\text { Incremento de la } \\
\text { ración pos-ayuno } \\
\text { no }\end{array}$ \\
\cline { 2 - 4 } & $\mathrm{N}^{\circ}$ de días & Días de ayuno & 0 \\
\hline Control (C) & 0 & Ninguno & 20 \\
Experimental (AY2) & 2 & Sábado y domingo & 20 \\
Experimental (AY3) & 3 & $\begin{array}{c}\text { Miércoles, sábado y } \\
\text { domingo }\end{array}$ & 20 \\
\hline
\end{tabular}

vestigación, se indica que dicha estrategia no es viable en el caso de cultivos intensivos (Shao et al., 2020), fundamentalmente por la alta incidencia de canibalismo en los individuos en cultivo (Stumpf et al., 2019); sin embargo, tal aseveración no sería aplicable en cultivos de menor densidad.

En esta investigación se ensayó la estrategia de ayuno y realimentación en periodos de cero, dos y tres días a la semana, con un incremento de la ración pos-ayuno de $20 \%$ en camarones juveniles cultivados en laboratorio a una densidad de 25 camarones $/ \mathrm{m}^{2}$ (equivalente a un cultivo semi-intensivo) y su efecto en el crecimiento, supervivencia, biomasa y conversión alimenticia; con el fin de demostrar la utilidad de dicha estrategia en el cultivo semi-intensivo de camarón blanco.

\section{Materiales y Métodos}

\section{Lugar del Estudio}

Se realizó en el Laboratorio de Acuicultura II de la Facultad de Ingeniería Pesquera y Ciencias del Mar, perteneciente a la Universidad Nacional de Tumbes, ubicada en la localidad de Puerto Pizarro, en la región Tumbes (Perú).

\section{Diseño Experimental}

El experimento siguió los lineamientos del diseño completamente aleatorizado, con tres tratamientos como se detallan en el Cuadro 1. Se utilizaron 100 camarones juveniles con peso promedio de $5.5 \pm 0.2 \mathrm{~g}$, provenientes de un campo de cultivo semi-intensivo de la zona. Los camarones fueron aclimatados en dos acuarios con $90 \mathrm{~L}$ de agua con salinidad de alrededor de 28\%. Luego del proceso de aclimatación, fueron transferidos a nueve acuarios conteniendo $90 \mathrm{~L}$ de agua salobre $(28.8 \pm 0.4 \%$ ) colocando ocho ejemplares por acuario, para tener una densidad de siembra de aproximadamente 25 camaro$\mathrm{nes} / \mathrm{m}^{2}$. Los acuarios fueron asignados aleatoriamente a los tratamientos, teniéndose tres acuarios por tratamiento.

Los acuarios permanecieron cubiertos con tela celosilla sujeta con elástico para evitar el escape de los camarones al saltar, así como para evitar la caída de insectos al agua. Además cada uno de ellos tuvo suministro permanente de aireación por medio de piedras difusoras, conectadas al sistema de aireación del laboratorio que fue impulsado por un aireador mecánico de $0.5 \mathrm{hp}$. 
Cuadro 2. Ajustes a la cantidad de alimento suministrado a diario en cada acuario

\begin{tabular}{ll}
\hline Alimento no consumido (\%) & $\begin{array}{l}\text { Ajuste }(\%) \text { en la cantidad de alimento a } \\
\text { suministrar }\end{array}$ \\
\hline Mayor a 25 & Reducir en 30 \\
Entre 25 y 10 & Reducir en 20 \\
Menor a 10 & Mantener \\
0 & Incrementar en 10 \\
\hline
\end{tabular}

La alimentación se realizó conforme al esquema establecido en su respectivo tratamiento. Se empleó alimento balanceado comercial con $35 \%$ de proteína, administrado dos veces al día (08:00 y 14:00 h). La cantidad de alimento (CA) suministrado por acuario fue establecida mediante la fórmula $\mathrm{CA}=$ $\mathrm{t} \times \mathrm{w} \times \mathrm{n} \times \mathrm{S}$, donde $\mathrm{t}$ es la tasa de alimentación propuesta por Talavera et al. (1998) y $\mathrm{w}, \mathrm{n}$ y $\mathrm{S}$ son el peso promedio, población inicial y supervivencia de los camarones del acuario, respectivamente. En los días posteriores, la cantidad de alimento a suministrar por día se fijó teniendo en consideración la cantidad de alimento no consumido durante el día en cada acuario, para lo cual se tomó lo criterios de ajuste que se muestran en el Cuadro 2, el cual está basado en la propuesta realizada por Talavera et al. (1998), con modificaciones.

Diariamente y luego de alimentar a los camarones, se realizó la limpieza del acuario, retirando los residuos de alimento, heces o exuvias de los camarones, mediante sifoneo. El nivel de agua del acuario se compensó luego de la limpieza, agregando agua con una salinidad adecuada para mantener relativamente estable la salinidad dentro del acuario. El experimento tuvo una duración de nueve semanas.

\section{Respuesta Productiva}

- El crecimiento evaluado como peso promedio de los camarones fue determinado una vez a la semana. Se calculó el incremento de peso semanal (IPS) con la fórmula IPS $=$ Wsac - Wsant, donde Wsac es el peso promedio de la semana actual y Wsant es el peso promedio de la semana anterior.

- La ganancia de peso porcentual (GPP) como GPP $=100 \times(\mathrm{Wf}-\mathrm{Wi}) / \mathrm{Wi}$, donde Wf es el peso promedio al final del experimento y Wi es el peso inicial.

- La supervivencia se determinó a diario. Se aplicó la fórmula $\mathrm{S} \%=\mathrm{n} / \mathrm{n}_{0} \times 100$, donde $\mathrm{S} \%$ es la supervivencia expresada como porcentaje, $\mathrm{n}$ es el número de ejemplares vivos $\mathrm{y}_{0}$ es el número de ejemplares al inicio del experimento.

- La biomasa de camarones por acuario se calculó semanalmente con la fórmula $\mathrm{B}=\Sigma \mathrm{Wi}$, donde $\mathrm{B}$ es la biomasa y Wi es el peso de cada camarón dentro del acuario.

- $\quad$ El factor de conversión relativo (FCR) para cada tratamiento se calculó como: $\mathrm{FCR}=\mathrm{AC} / \mathrm{B}$, donde $\mathrm{AC}$ es el peso de alimento consumido acumulado y $\mathrm{B}$ es la biomasa de camarones en dicho tratamiento. 
Cuadro 3. Incremento de peso (g) de camarones Litopenaeus vannamei según tratamiento (promedio \pm desviación estándar)

\begin{tabular}{lccc}
\hline & \multicolumn{3}{c}{ Tratamiento } \\
\cline { 2 - 4 } & Control & AY2 & AY3 \\
\hline Peso inicial $(\mathrm{g})$ & $5.43 \pm 0.27^{\mathrm{a}}$ & $5.49 \pm 0.33^{\mathrm{a}}$ & $5.50 \pm 0.17^{\mathrm{a}}$ \\
Peso final (g) & $13.39 \pm 0.22^{\mathrm{a}}$ & $12.97 \pm 0.29^{\mathrm{a}}$ & $11.93 \pm 0.13^{\mathrm{b}}$ \\
Incremento de peso semanal (g) & $0.83 \pm 0.06^{\mathrm{a}}$ & $0.78 \pm 0.02^{\mathrm{a}}$ & $0.67 \pm 0.01^{\mathrm{a}}$ \\
Ganancia de peso porcentual (\%) & $7.96 \pm 0.08^{\mathrm{a}}$ & $7.48 \pm 0.05^{\mathrm{a}}$ & $6.43 \pm 0.05^{\mathrm{b}}$ \\
Supervivencia (\%) & $100 \pm 0^{\mathrm{a}}$ & $100 \pm 0^{\mathrm{a}}$ & $100 \pm 0^{\mathrm{a}}$ \\
\hline
\end{tabular}

$a, b$ Superíndices diferentes dentro de líneas indican diferencias significativas $(p<0.05)$

AY2 y AY3: Ayuno de dos o tres días por semana con $20 \%$ de incremento de la ración posayuno

- Adicionalmente, los parámetros físicos químicos de calidad de agua de cada acuario se midieron cada semana.

\section{Análisis Estadístico}

Los datos obtenidos sobre crecimiento, supervivencia, biomasa, alimento consumido, FCR y parámetros de calidad de agua de cada uno de los tratamientos fueron analizados para determinar si existieron diferencias significativas entre ellos, utilizando análisis de varianza de una sola vía. Cuando se determinó la existencia de diferencia significativa se realizó la prueba de rangos múltiples de Duncan. Ambas pruebas se evaluaron con 0.05 de nivel de significancia.

\section{Resultados y Discusión}

\section{Crecimiento y Supervivencia}

El peso inicial de los camarones fue similar entre tratamientos (Cuadro 3). Se pudo determinar que conforme se fue desarrollando el cultivo el crecimiento de los camarones que tuvieron dos días de ayuno (AY2) se mantuvo similar al del grupo control, en tanto que los camarones que tuvieron tres días de ayuno a la semana mantuvieron un peso promedio significativamente menor al grupo control y AY2 ( $<<0.05)$ (Cuadro 4).

Al final del experimento, los pesos promedios de los camarones de los tratamientos control y AY2 fueron $13.39 \pm 0.22 \mathrm{~g}$ y 12.97 $\pm 0.29 \mathrm{~g}$ respectivamente, sin diferencia significativa entre ellos, pero superiores al peso logrado por los camarones en el tratamiento AY3 ( $\mathrm{p}<0.05$ ) (Cuadro 3). Resultados similares se observaron para el incremento de peso semanal y la ganancia de peso porcentual (GPP) (Cuadro 3).

El crecimiento de los camarones $L$. vannamei no se vio afectado cuando se tuvo un ayuno de dos días. Este resultado es congruente con experiencias previas de otros investigadores que también observaron que el crecimiento no se vio afectado por ayunos de corta duración. Así, Shao et al. (2020) encontraron que no se afectó el peso final, el incremento de peso y la GPP al someter a ejemplares de L. vannamei a uno o dos días 
Cuadro 4. Crecimiento en peso promedio (g) de camarones Litopenaeus vannamei según tratamiento (promedio \pm desviación estándar)

\begin{tabular}{cccc}
\hline \multirow{3}{*}{$\begin{array}{c}\text { Semana de } \\
\text { cultivo }\end{array}$} & Control & AY2 & AY3 \\
\cline { 2 - 4 } & $5.43 \pm 0.27^{\mathrm{a}}$ & $5.49 \pm 0.33^{\mathrm{a}}$ & $5.50 \pm 0.17^{\mathrm{a}}$ \\
1 & $6.26 \pm 0.25^{\mathrm{a}}$ & $6.27 \pm 0.34^{\mathrm{a}}$ & $6.17 \pm 0.16^{\mathrm{a}}$ \\
2 & $7.13 \pm 0.25^{\mathrm{a}}$ & $7.07 \pm 0.38^{\mathrm{a}}$ & $6.86 \pm 0.14^{\mathrm{a}}$ \\
3 & $8.03 \pm 0.27^{\mathrm{a}}$ & $7.91 \pm 0.36^{\mathrm{a}}$ & $7.56 \pm 0.13^{\mathrm{a}}$ \\
4 & $8.91 \pm 0.25^{\mathrm{a}}$ & $8.73 \pm 0.33^{\mathrm{ab}}$ & $8.29 \pm 0.12^{\mathrm{b}}$ \\
5 & $9.80 \pm 0.25^{\mathrm{a}}$ & $9.59 \pm 0.32^{\mathrm{a}}$ & $9.02 \pm 0.10^{\mathrm{b}}$ \\
6 & $10.68 \pm 0.23^{\mathrm{a}}$ & $10.45 \pm 0.34^{\mathrm{a}}$ & $9.76 \pm 0.12^{\mathrm{b}}$ \\
7 & $11.59 \pm 0.23^{\mathrm{a}}$ & $11.29 \pm 0.32^{\mathrm{a}}$ & $10.51 \pm 0.12^{\mathrm{b}}$ \\
8 & $12.48 \pm 0.23^{\mathrm{a}}$ & $12.14 \pm 0.31^{\mathrm{a}}$ & $11.22 \pm 0.13^{\mathrm{b}}$ \\
9 & $13.39 \pm 0.22^{\mathrm{a}}$ & $12.97 \pm 0.29^{\mathrm{a}}$ & $11.93 \pm 0.13^{\mathrm{b}}$ \\
\hline
\end{tabular}

$a, b$ Superíndices diferentes dentro de líneas indican diferencias significativas $(p<0.05)$

AY2 y AY3: Ayuno de dos o tres días por semana con $20 \%$ de incremento de la ración posayuno

de ayuno por semana; de igual manera Bezerra et al. (2019) encontraron que dos días de ayuno permitió un crecimiento similar al de camarones control, demostrando que ayunos cortos no afectan el crecimiento del camarón. Esto mismo fue demostrado por Utomo et al. (2019) con crustáceos Macrobrachium rosenbergii, el cual tampoco afectó su crecimiento cuando fue sometido a esquemas de 1 y 2 días de ayuno.

La supervivencia de los camarones no fue afectada en esta investigación, llegando todos los camarones a sobrevivir en los tres tratamientos. Este resultado contrasta con el estudio de Shao et al. (2020), quienes encontraron menor supervivencia en los grupos con ayuno, resultado que se explica por el empleo de una densidad de 200 camarones $/ \mathrm{m}^{2}$, equivalente a la de un cultivo intensivo. Es un hecho conocido que las mayores densidades de cultivo generan conductas agresivas y territoriales e incluso canibalismo (Krumme- nauer et al., 2011; Esparza-Leal et al., 2020; Fleckenstein et al., 2020).

Supervivencias elevadas ante periodos cortos de ayuno se han reportado en experimentos con otros crustáceos como por ejemplo en Fenneropenaeus chinensis, en los que se registraron supervivencias de $99.3 \%$ en ensayos de seis días de ayuno seguidos de 30 días de realimentación (Wu y Dong, 2002); así como con Cherax quadricarinatus donde se logró una supervivencia de $96 \%$ al ser sometido por 45 días a ciclos de cuatro días de ayuno por cuatro días de realimentación (Stumpf y López, 2015).

El hecho de que se puedan lograr altos niveles de supervivencia en estos ensayos se explica, según Maciel et al. (2018) y Yildirim y Aktaç33 (2019) a que el ayuno en los crustáceos es un aspecto que se produce rutinaria-mente en la naturaleza, pues por diversas razones dichos organismos no siempre pue- 
Cuadro 5. Biomasa, consumo de alimento y factor de conversión relativo de camarones Litopenaeus vannamei según tratamiento (promedio \pm desviación estándar)

\begin{tabular}{lrrr}
\hline & \multicolumn{3}{c}{ Tratamiento } \\
\cline { 2 - 4 } & \multicolumn{1}{c}{ Control } & \multicolumn{1}{c}{ AY2 } & \multicolumn{1}{c}{ AY3 } \\
\hline Biomasa final $(\mathrm{g})$ & $107.15 \pm 1.76^{\mathrm{a}}$ & $103.76 \pm 2.31^{\mathrm{a}}$ & $95.41 \pm 1.04^{\mathrm{b}}$ \\
Incremento de biomasa $(\mathrm{g})$ & $63.68 \pm 0.65^{\mathrm{a}}$ & $59.81 \pm 0.39^{\mathrm{b}}$ & $51.41 \pm 0.40^{\mathrm{c}}$ \\
Consumo de alimento $(\mathrm{g})$ & $96.84 \pm 1.81^{\mathrm{a}}$ & $70.29 \pm 1.22^{\mathrm{b}}$ & $56.95 \pm 0.38^{\mathrm{c}}$ \\
Factor de conversión relativo & $1.52 \pm 0.06^{\mathrm{a}}$ & $1.18 \pm 0.09^{\mathrm{b}}$ & $1.11 \pm 0.11^{\mathrm{c}}$ \\
\hline a,b,c Superíndices diferentes dentro de líneas indican diferencias significativas ( $\mathrm{p}<0.05$ ) \\
AY2 y AY3: Ayuno de dos o tres días por semana con 20\% de incremento de la ración pos- \\
ayuno
\end{tabular}

den conseguir alimento y, por lo tanto, presentan mecanismos bioquímicos que les permiten adaptarse a soportar incluso lapsos largos de ayuno. Así, ayunos de corta duración no deberían representar una amenaza mayor a su supervivencia.

\section{Biomasa y Consumo de Alimento}

La biomasa de camarones al final del experimento fue estadísticamente similar entre el tratamiento control y el de dos días de ayuno a la semana, pero inferior en el caso del tratamiento con tres días de ayuno (Cuadro 5). El consumo de alimento significativamente fue más alto en el tratamiento control $(96.84 \pm 1.81 \mathrm{~g})$ y menor con relación al número de días de ayuno $(70.29 \pm 1.22 \mathrm{~g}$ para dos días y $56.95 \pm 0.38 \mathrm{~g}$ para tres días de ayuno semanales) ( $\mathrm{p}<0.05$; Cuadro 5). Estos resultados concuerdan con los reportados por Bezerra et al. (2019) y Cota (2020).

Se obtuvo un ahorro de $27.4 \%$ de alimento con dos días de ayuno y de $41.2 \%$ con tres días de ayuno; sin embargo, tres días de ayuno tuvieron un efecto negativo en el crecimiento, por lo que el tratamiento más viable para ahorrar alimento fue aquel que tuvo dos días de ayuno a la semana. El ahorro de alimento obtenido es similar a la cantidad que Fóes et al. (2016) señalaron como probable para un cultivo de L. vannamei que aproveche el crecimiento compensatorio desarrollado luego de un periodo de privación alimenticia corta seguida de realimentación.

\section{Factor de Conversión Relativo}

El factor de conversión relativo (FCR) para los tratamientos control, y los que tuvieron dos y tres días de ayuno fue de 1.52, 1.18 y 1.11 , respectivamente, siendo estadísticamente diferentes entre ellos ( $p<0.05$; Cuadro 5). Dado que el FCR es un indicador de la eficiencia en la conversión de alimento en masa corporal, es de esperar que los camarones que evidenciaron crecimiento compensatorio tengan mejores valores de FCR (Yildirim y Aktaç, 2019; Shao et al., 2020). En este sentido, Bezerra et al. (2019) reportaron una mejora en el FCR cuando se tuvo un ayuno corto (un día de alimentación por dos días de ayuno), pero no cuando se tuvo un ayuno más prolongado (un día de alimentación por cuatro de ayuno), por lo que se 
Cuadro 6. Parámetros fisicoquímicos evaluados en el agua de cultivo de camarones Litopenaeus vannamei según tratamiento (promedio \pm desviación estándar)

\begin{tabular}{lrrr}
\hline \multirow{2}{*}{ Parámetros } & \multicolumn{3}{c}{ Tratamiento } \\
\cline { 2 - 4 } & \multicolumn{1}{c}{ Control } & \multicolumn{1}{c}{ AY2 } & \multicolumn{1}{c}{ AY3 } \\
\hline Temperatura $\left({ }^{\circ} \mathrm{C}\right)$ & $28.8 \pm 0.5^{\mathrm{a}}$ & $28.8 \pm 0.6^{\mathrm{a}}$ & $28.9 \pm 0.5^{\mathrm{a}}$ \\
$\mathrm{pH}$ & $7.92 \pm 0.14^{\mathrm{a}}$ & $7.82 \pm 0.15^{\mathrm{b}}$ & $7.87 \pm 0.12^{\mathrm{ab}}$ \\
Salinidad $(\mathrm{g} / \mathrm{l})$ & $29.7 \pm 0.9^{\mathrm{a}}$ & $29.6 \pm 1.1^{\mathrm{a}}$ & $29.8 \pm 1.0^{\mathrm{a}}$ \\
\hline
\end{tabular}

$a, b$ Superíndices diferentes dentro de líneas indican diferencias significativas $(p<0.05)$

AY2 y AY3: Ayuno de dos o tres días por semana con $20 \%$ de incremento de la ración posayuno

puede concluir que es posible mejorar el FCR cuando se hace uso de ayunos cortos.

\section{Parámetros Fisicoquímicos del Agua}

No se observaron diferencias significativas en los parámetros fisicoquímicos del agua de los acuarios, a excepción del pH, aunque la diferencia fue mínima (Cuadro 6). Los parámetros observados se hallaron dentro de los rangos de calidad de agua recomendados para el cultivo de camarón blanco por Van Wik y Scarpa (1999).

\section{Conclusiones}

- El ayuno de dos días a la semana con un incremento de $20 \%$ de la ración pos-ayuno origina crecimiento compensatorio en el camarón cultivado a densidad de 25 individuos $/ \mathrm{m}^{2}$, lo que le permite mantener un peso y biomasa estadísticamente similar al de los camarones que no tuvieron ayuno.

- Se logró un ahorro de $27.4 \%$ en el alimento suministrado y una mejora significativa en el factor de conversión relativo - FCR (1.18 en camarones con dos días de ayuno frente a 1.52 en camarones sin ayuno).
- Los camarones con tres días de ayuno a la semana tuvieron un peso promedio y biomasa significativamente inferior, pero mostraron un mayor ahorro en el consumo de alimento y un mejor FCR.

- La supervivencia no se vio afectada con el número de días de ayuno.

- El tratamiento con ayuno de dos días a la semana e incremento de $20 \%$ de la ración pos-ayuno es una alternativa viable para el cultivo de Litopenaeus vannamei en densidad equivalente a la de un cultivo semi-intensivo.

\section{Literatura Citada}

1. Bardera G, Owen MAG, Façanha FN, Alcaraz-Calero JM, Alexander ME, Sloman KA. 2021. The influence of density and dominance on Pacific white shrimp (Litopenaeus vannamei) feeding behaviour. Aquaculture 531: 735949. doi: 10.1016/j.aquaculture.2020.735949

2. Bezerra D, Dias CD, Soares C. 2019. Performance of shrimp Litopenaeus vanname $i$ submitted to different fasting periods. Braz J Anim Environ Res 2: 1074-1081.

3. Cota MJ. 2020. Genes asociados al crecimiento compensatorio en el camarón blanco del Pacífico Penaeus vannamei. 
Tesis de Maestría. México: Centro de Investigaciones Biológicas del Noroeste, SC. $86 \mathrm{p}$.

4. Esparza-Leal HM, Ponce-Palafox JT, Álvarez-Ruiz, P, López-Álvarez ES, Vázquez-Montoya N, López-Espinoza M, Mejia MM, et al. 2020. Effect of stocking density and water exchange on performance and stress tolerance to low and high salinity by Litopenaeus vannamei postlarvae reared with biofloc in intensive nursery phase. Aquacult Int 28: 1473-1483. doi: 10.1007/s10499-02000535-y

5. Faillace, JF, Vergara R, Suarez A. 2016. Evaluación de una fórmula alimenticia para camarón de cultivo ( $L$. vannamei) con inclusión de proteína vegetal a base de harina de soya. Revista AquaTIC 44: 12-29.

6. [FAO] Food and Agricultural Organization of the United Nations. 2018. Fishery statistical collections. global production. Rome: FAO. [Internet]. Available in: http://www.fao.org/fishery/ statistics/global-production/en

7. [FAO] Organización de las Naciones Unidas para la Alimentación y la Agricultura. 2020. El estado mundial de la pesca y la acuicutura 2020: La sostenibilidad en acción. Roma: FAO. 243 p. [Internet]. Disponible en: http:// www.fao.org/3/ca9229es/CA9229ES.pdf

8. Fleckenstein LJ, Kring NA, Tierney TW, Fisk JC, Lawson BC, Ray AJ. 2020. The effects of artificial substrate and stocking density on Pacific white shrimp (Litopenaeus vannamei) performance and water quality dynamics in high tunnel-based biofloc systems Aquacult Eng 90: 102093. doi: 10.1016/ j.aquaeng.2020.102093

9. Fóes G, Krummenauer D, Lara G, Poersch L, Wasielesky WJ. 2016. Long term storage and the compensatory growth of white shrimp Litopenaeus vannamei in aquaculture ponds. Lat Am J Aquat Res 44: 588-594. doi: 10.3856/ vol44-issue3-fulltext-17

10. Gamboa-Delgado J, Nieto-López MG, Maldonado-Muñiz M, VillarrealCavazos D, Tapia-Salazar M, CruzSuárez LE. 2020. Comparing the assimilation of dietary nitrogen supplied by animal-, plant- and microbial-derived ingredients in Pacific white shrimp Litopenaeus vannamei: A stable isotope study. Aquac Rep 17: 100294. doi: 10.1016/j.aqrep.2020.100294

11. Ghosh AK. 2018. Effect of feeding level on growth, body composition, fatty acid profile, and nutrient accumulation in shrimp (Litopenaeus vannamei). Aquacult Int 26: 405-417. doi: 10.1007/ s10499-017-0225-z

12. Ghosh AK, Biswas M, Azad MAK, Saiful S, Islam KAH, Shah MS. 2018. Effect of restricted feeding on growth performance and feed utilization of freshwater prawn (Macrobrachium rosenbergii) in pond aquaculture system. Khulna Univ Studies 15: 13-22.

13. Hamidoghli A, Won S, Farris NW, Bae J, Choi W, Yun H, Bai SC. 2020. Solid state fermented plant protein sources as fish meal replacers in whiteleg shrimp Litopaeneus vannamei. Anim Feed Sci Tech 264: 114474. doi: 10.1016/ j.anifeedsci.2020.114474

14. Krummenauer D, Peixoto S, Cavalli RO, Poersch LH, Wasielesky W. 2011. Superintensive culture of white shrimp, Litopenaeus vannamei, in a biofloc technology system in Southern Brazil at different stocking densities. J World Aquacult Soc 42: 726-733. doi: 10.1111/ j.1749-7345.2011.00507.x

15. Lara G, Hostins B, Bezerra A, Poersch L, Wasielesky W. 2017. The effects of different feeding rates and re-feeding of Litopenaeus vannamei in a biofloc culture system. Aquacult Eng 77: 20-26. doi: 10.1016/j.aquaeng.2017.02.003 
16. Liu W, Lu X, Jiang $M, W u F$, Tian J, Yang C, Yu L, Wen H. 2018. Effects of dietary manipulation on compensatory growth of juvenile genetically improved farmed tilapia (Oreochromis niloticus). Fish Physiol Biochem 45: 21-32. doi: 10.1007/s10695-018-0531-z

17. Maciel JC, Francisco CJ, MirandaFilho KC. 2018. Compensatory growth and feed restriction in marine shrimp production, with emphasis on biofloc technology. Aquacult Int 26: 203-212. doi: 10.1007/s10499-017-0209-z

18. Martínez LR, Martínez M, Miranda A, López JA. 2013. Selección de alimentos y estrategias de alimentación acordes a las condiciones de cultivo del camarón. En: Cruz LE, Ricque D, Tapia $\mathrm{M}$, et al. (eds). Contribuciones recientes en alimentación y nutrición acuícola. Monterrey: Univ. Autónoma de Nuevo León. p 84-105.

19. Mohanty RK, Mohapatra A. 2017. Cyclic feed restriction on growth compensation of Penaeus monodon (Fabricius): science meets practice. Indian J Geo-Mar Sci 46: 2008-2016

20. Mohanty $R$, Thakur A, Nayak A, Ambast S. 2020. Compensatory growth response and feed intake pattern of Indian major carps in confined aquatic environment. J Ind Soc Coastal Agric Res 38: 43-49.

21. [Produce] Ministerio de la Producción. 2018. Anuario estadístico pesquero y acuícola 2017. Lima: Produce. 205 p. [Internet]. Disponible en: http:// ogeiee.produce.gob.pe/images/Anuario/ Pesca_2017.pdf

22. Rodríguez-González T, Valverde J, Sykes A, García B. 2018. Common octopus (Octopus vulgaris) performance when including fasting on feeding schemes: preliminary data regarding a formulated feed. Adv Res 13: 1-11. doi: 10.9734/AIR/2018/39175

23. Savoie A, Le François NR, Lamarre SG, Dupuis F, Blier PU. 2017. Preliminary investigations of the physiolo- gical adjustments associated with compensatory growth in juvenile brook charr (Salvelinus fontinalis). J Appl Aquac 29: 16-32. doi: 10.1080/ 10454438.2016.1269531

24. Shao J-C, Zhu W-Q, Liu M, Wang L, Zhao W. 2020. Cyclical fasting and refeeding is not an advisable feeding strategy for white shrimp (Penaeus vannamei). Aquac Rep 18: 100429. doi: 10.1016/j.aqrep.2020.100429

25. Steinberg CEW. 2018. Dietary restriction, starvation, compensatory growth 'short-term fasting does not kill you: It can make you stronger'. In: Steinberg CEW (ed). Aquatic animal nutrition. Springer. p 137-287.

26. Stumpf L, López LS. 2015. Compensatory growth in juveniles of freshwater redclaw crayfish Cherax quadricarinatus reared at three different temperatures: hyperphagia and food efficiency as primary mechanisms. Plos One 10: e0139372. doi: 10.1371/ journal.pone. 0139372

27. Stumpf L, Sarmiento PN, Timpanaro S, López L. 2019. Feasibility of compensatory growth in early juveniles of «red claw» crayfish Cherax quadricarinatus under high density conditions. Aquaculture 510: 302-310. doi: 10.1016/j.aquaculture.2019.05.053

28. Talavera V, Sánchez D, Zapata LM. 1998. Métodos de alimentación. Bol Nicovita Camarón de Mar 3: 1-6.

29. Tarazona U, León J, Galindo N, Vallejo M, Marguet E. 2018. Caracterización de actinomicetos de sedimento marino y su actividad antagonista frente a Vibrio sp aislados de «langostino blanco» Litopenaeus vannamei (Boone, 1931). Rev Inv Vet Perú 29: 676-691. doi: 10.15381/rivep.v29i2.14999

30. Ullman C, Rhodes MA, Allen D. 2019. Feed management and the use of automatic feeders in the pond production of Pacific white shrimp Litopenaeus vannamei. Aquaculture 498: 44-49. doi: 10.1016/j.aquaculture.2018.08.040 
31. Utomo WD, Hakim RR, Sutarjo GA. 2019. Effect of periodic fasting on growth and feed efficiency in giant freshwater prawn (Macrobrachium rosenbergii, de Man 1879). Indonesian J Trop Aquatic 2: 53-58. doi: 10.22219/ ijota.v2i2.6679

32. Van Wik P, Scarpa J. 1999. Water quality requirements and management. In: Van Wyk P, Davis-Hodgkins M, Laramore $\mathrm{R}$, et al. (eds). Farming marine shrimp in recirculating freshwater systems. Tallahassee: Florida Department of Agriculture and Consumer Services. p 141-162.

33. Wu L, Dong S. 2002. Compensatory growth responses in juvenile Chinese- shrimp, Fenneropenaeus chinensis, at different temperatures. J Crustacean Biol 22: 511-520. doi: 10.1163/2002197599990262

34. Yildirim M, Aktaç M. 2019. Could the growth of Litopenaeus vannamei be compensated by long-term cyclic feed deprivation and following refeeding? J Appl Aquac 31: 224-235. doi: 10.1080/ 10454438.2018.1536007

35. Zhu Z-M, Lin X-T, Pan J-X, Xu Z-N. 2016. Effect of cyclical feeding on compensatory growth, nitrogen and phosphorus budgets in juvenile Litopenaeus vannamei. Aquac Res 47: 283-289. doi: 10.1111/are.12490 